\title{
The Graph Representation Approach to Topological Field Theory in $2+1$ Dimensions
}

\author{
Stephen P. Martin \\ Department of Physics \\ University of Florida \\ Gainesville, FL 32611
}

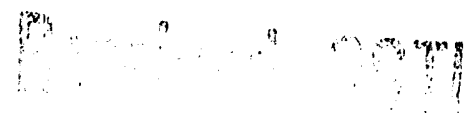

ABSTRACT: An alternaive definition of topological quantum field theory in $2+1$ dimensions is discussed. The fundamental objects in this approach are not gauge fields as in the usual approach, but non-local observables associated with graphs. The classical theory of graphs is defined by postulating a simple diagrammatic rule for computing the Poisson bracket of any two graphs. The theory is quantized by exhibiting a quantum deformation of the classical Poisson bracket algebra, which is realized as a commutator algebra on a Hilbert space of states. The wavefunctions in this "graph representation" approach are functionals on an appropriate set of graphs. This is in contrast to the usual "connection representation" approach in which the theory is defined in terms of a gauge field and the wavefunctions are functionals on the space of flat spatial connections modulo gauge transformations.

\section{DISCLAIMER}

This report was prepared as an account of work sponsored by an agency of the United States Government. Neither the United States Government nor any agency thereof, nor any of their employees, makes any warranty, express or implied, or assumes any legal liability or responsibility for the accuracy, completeness, or usefulness of any information, apparatus, product, or process disclosed, or represents that its use would not infringe privately owned rights. Refer ence herein to any specific commercial product, process, or service by trade name, trademark, manufacturer, or otherwise does not necessarily constitute or impiy its endorsemeni, Iecininmendation, or favoring by the United States Government or any agency thereof. and opinions of authors expressed herein do not necessarily state or reflect those of the United States Government or any agency thereof. 


\section{Introduction}

One of the most significant truths in fundamental physics is that successful physical models are based on local field theories blessed with local gauge invariances. One ramification of a theory possessing such local symmetries is that it is defined in terms of variables which are in some sense "mostly fake". By this we mean, for example, that the gauge field for a given single physical configuration in Yang-Mills theory could take on, by virtue of local gauge invariance, an uncountable infinitude of clifferent values. Of course, one can identify sets of observables which completely specify the physical configuration and are yet invariant under gauge transformations. Under favorable conditions, one example of such a set of observables are the S-matrix elements. A more appropriate class of observables for our present discussion is the set of Wilson loops. If the fundamental gauge field $A_{\mu}$ takes values in the Lie algebra of some Lie group $\mathcal{G}$ and is subject to a gauge transformation

$$
A_{\mu} \rightarrow g^{-1} A_{\mu} g+g^{-1} \partial_{\mu} g
$$

then one has observables

$$
W_{R}(L)=\operatorname{Tr}_{R} P \exp \oint_{L} d x^{\mu} A_{\mu}
$$

where the trace is taken over an arbitrary representation $R$ of $\mathcal{G}$, and $L$ may be any closed loop. Furthermore, it can be shown that in some sense, the Wilson loop observables capture all of the gauge invariant, "non-fake" information present in the gauge field $A_{\mu}$. This property of the Wilson loop observables for gauge fields is entirely kinematical, and has nothing to do with whatever dynamics governs the theory, as long as it respects the gauge symmetry (1.1).

In view of this, it is tempting to adopt the following view. The Wilson loops, in the usual approach to gauge theories, are derived quantities which are determined as functionals of the gauge field $A_{\mu}$. However, one might attempt to define the theory directly in terms of loop observables $W_{R}(L)$ which are now taken to be independent variables, instead of being defined by (1.2). The definition of the theory would then consist of (i) a set of rules governing the dynamics of the $W_{R}(L)$, and (ii) a set of "kinematical" rules 
governing the redundancy of the $W_{R}(L)$. At least in some appropriate physical limit, one should require that the theory defined by these rules will reproduce the kinematical and dynamical properties of the Wilson loops as derived quantities in the corresponding theory defined in terms of gauge fields. (This approach is similar in spirit to the one proposed lorig ago by Mandelstam, who showed in some early papers [1] that it is possible to define Yang-Mills theory and gravity in $3+1$ dimensions without gauge potentials or metric fields, by using path-ordered exponentials.)

It turns o'st to be a convenient simplification to define the theory in terms of a closely related set of observables called Wilson graphs, of which the Wilson loops are a subset. The Wilson graphs are formed by taking path-ordered exponentials of the gauge field along segments which are connected to each other at their mutual endpoints. The path-ordered exponential segments may each be in an arbitrary representation of $\mathcal{G}$. Where $n$ pathordiered exponentials in the representations $R_{1}, R_{2}, \ldots, R_{n}$ of $\mathcal{G}$ join at a vertex point, they are coupled by an invariant symbol of $\mathcal{G}$ denoted by $[V]^{i_{1} \ldots i_{n}}$; the indices $i_{1} \ldots i_{n}$ correspond to the representations $R_{1} \ldots R_{n}$ respectively. There is an invariant symbol $[V]^{i_{1} \ldots i_{n}}$ for each occurrence of the trivial (singlet) representation of $\mathcal{G}$ in the direct sum decomposition of the direct product $R_{1} \otimes \ldots \otimes R_{n}$. The defining property of the invariant symbol is that the total effect of a $\mathcal{G}$-rotation on each of the indices of $[V]^{i_{1} \ldots i_{n}}$ is to leave it unchanged; this ensures that the effects of the gauge transformations on the path-ordered exponential segments running into the vertex will cancel. Thus the Wilson graphs are gauge invariant. Each segment of the graph is labeled by a representation of $\mathcal{G}$ and each vertex of the graph is labeled by an invariant symbol of $\mathcal{G}$. Segments which are labeled by complex representations of $\mathcal{G}$ must also carry an arrow to label their orientation. Note that the product of any two Wilson graphs is clearly another Wilson graph, since once can always consider the two graphs to be joined by a segment in the trivial representation.

In this paper, we will show that the program of defining a gauge theory directly in terms of non-local observables can indeed be carried to a successful conclusion in the particular special case of topological Chern-Simons gauge theory in three dimensions. Of course, the quantization of this theory has already been worked out, especially from the work of Witten 
[2-5], in which the relevance of topological gauge theory to gravity in three dimensions, knot theory, quantum groups and lattice statistical mechanics in two dimensions were explored. Witten showed that Chern-Simons gauge theory could be solved exactly by using a formal correspondence between the three-dimensional theory and two-dimensional conformal field theory. From this one can distill a few diagrammatic rules which allow one to calculate arbitrary expectation values of the observables. Once this is established, one way to proceed (which we will not adopt here) would be to adopt these diagrammatic rules as the definition of the topological theory. Our purpose here is instead to show how the program of understanding the theory from a definition? directly in terms of non-local observables is accomplished, starting from a classical canonical approach. One can make a rigorous definition of a classical "connection" theory, defined in terms of a gauge field, and a "graph theory" defined directly in terms of the graphs, and conspire to make them agree on their classical predictions regarding physical observables. We then proceed to describe the direct quantization of the theory of graphs.

\section{Classical Topological Gauge Theory as a Theory of Graphs}

The action for topological Chern-Simons gauge theory on a three manifold $\mathcal{M}$ is

$$
S_{C S}=\frac{k}{4 \pi} \operatorname{Tr} \int_{\mathcal{M}} \epsilon^{\mu \nu \rho}\left[A_{\mu} \partial_{\nu} A_{\rho}+\frac{2}{3} A_{\mu} A_{\nu} A_{\rho}\right]
$$

where the trace is taken in the defining representation of $\mathcal{G}$ and the coupling constant $k$ must be an integer. This action is invariant under the gauge transformation (1.1). In order to understand the canonical structure of the theory one considers the three-manifold to be split into a two-dimensional space $\Sigma$ and time. Then the action can be rewritten as

$$
S_{C S}=\frac{k}{8 \pi} \operatorname{Tr} \int \mathrm{d} t \int_{\Sigma} \epsilon^{\mu \nu}\left[-A_{\mu} \partial_{t} A_{\nu}+A_{t} F_{\mu \nu}\right]
$$

where $F_{\mu \nu}=\partial_{\mu} A_{\nu}-\partial_{\nu} A_{\mu}+\left[A_{\mu}, A_{\nu}\right]$ is the field strength associated with the spatial parts of the gauge field. Let us write $A_{\mu}=A_{\mu}^{a} T_{a}$ where the $T_{a}$ are the generators of $\mathcal{G}$ in the defining representation. Then we can read off the Poisson brackets of the gauge field

$$
\left\{A_{\mu}^{a}(x), A_{\nu}^{b}(y)\right\}_{P . B .}=\frac{4 \pi}{k} \epsilon_{\mu \nu} \delta^{a b} \delta^{(2)}(x-y)
$$


The time component of the gauge field $A_{t}$ just becomes a Lagrange multiplier enforcing the canonical constraint

$$
F_{\mu \nu}=0
$$

where now the vector indices $\mu$ and $\nu$ are spacelike. This constraint (2.3) requires that, the spatial part of the gauge field is locally flat. In terms of the Wilson graph observables, we can then make the following statements. First, the Wilson graphs which are restricted to the spatial hypersurface $\Sigma$ give a complete and redundant set of coordinates on the reduced phase space. Second, because the spatial part of the gauge field is locally flat, any spatial Wilson graph observable can be smoothly deformed in $\Sigma$ without changing it. This means that for Chern-Simons gauge theory, one only needs to consider equivalence classes of spatial Wilson graphs which can be smoothly deformed into each other. Thus for topological gauge theory, there are only a countably infinite number of distinct Wilson graph observables.

The set of rules governing the "dynamics" of the TVilson graphs will consist of a Hamiltonian, defined as a functional of the Wilson graphs, and a rule for computing the Poisson bracket of any pair of Wilson graphs. The easy part of this is the Hamiltonian, which generates cime evolution. In the canonical approach to a topological theory, all observables are time independent, so the Hamiltonian can simply be taken to be zero. However, there are still non-trivial dynamics in the sense that there are non-trivial Poisson brackets between certain pairs of observables. Let us schematically label the Wilson graphs e.g. $G_{A}$ with the single index $A$ representing all of the data needed to specify the graph, namely which representations of $\mathcal{G}$ are present on the segments, and which invariant symbols are used at the vertices, and the topological relationship between the graph and the 2-manifold $\Sigma$ on which the graph lives in the canonical approach. Then the Poisson bracket aigebra of the classical theory just takes the form

$$
\left\{G_{A}, G_{B}\right\}_{P . B .}=\frac{1}{k} \sum_{C} F_{A B}^{C} G_{C}
$$

with structure constants $F_{A B}^{C}$ which we need to specify. Onc can cierive this Poisson bracket algebra from the Poisson brackets of the gauge field (2.2). Before loing this, let us 
first make some rather simple qualitative observations. First, we note that if two Wilson graphs can be smoothly deformed in $\Sigma$ so that they do not intersect, then their Poisson bracket must vanish. This follows immediately from the locality of the Poisson bracket of the gauge field (2.2) and the fact that the Wilson graphs contain no derivatives of the gauge field. For example, the two Wilson graphs on the genus 2 surface $\Sigma$ in Fig. 1 must have zero Poisson bracket since they do not intersect. Furthermore, we see immediately that any Wilson graph which can be deformed to a point (i.e. does not "encircle" some topological disturbance on $\Sigma$ ) can be set equal to a numerical constant, since it clearly has vanishing Poisson bracket with every observable. To evaluate the constant number that the graph is equal to, one can simply set each segment in the graph equal to the identity matrix in the appropriate representation, since locally the flat gauge field $A_{\mu}$ can always be set equal to zero. Thus e.g. a Wilson loop in a representation $R$ which does not enclose any handle or puncture in $\Sigma$ is classically just equal to the dimension of $R$.

We will return later to complete the ciscussion of the dynamics by actually computing the structure constants in the Poisson bracket algebra (2.4). But for now let us proceed to the discussion of the set of kinematical rules governing the redundancy of the Wilson graphs in Chern-Simons gauge theory. Since the product of any two Wilson graphs is always another, all such redundancy relations can be written as the vanishing of certain special linear combinations of graphs. Schematically, we have

$$
\sum_{A} l_{i}^{A} G_{A}=0
$$

(The index $i$ on the structure constants $l_{i}^{A}$ just labels which of the redundancy relations we are considering.)

Incidentally, we should mention why one uses Wilson graphs rather than Wilson loops to define the theory. After all, one might argue that the Wilson loops are a complete set of observables, in the sense that all physical observables can be written in terms of them. So why not just quantize a theory of loops? In principle this can be done, but in the most general case it is much more complicated. The point is that the Poisson bracket of two Wilson loops is, in general, not even a polynomial in loops, but it can always be 
written as a linear combination of graph observables. (For some groups, like $S U(2)$ or the Poincare group in $2+1$ dimensions, it is actually easy to just use loop observables in the fundamental representations which can wind around each handle more than once, but this is by no means a general feature of all Lie groups.)

The redundancy rules (2.5) just follow from the fact that Wilson graphs can be smoothly deformed and from a little bit of group theory. It is useful first to remark that every graph can be written just as a sum of graphs which contain at most 3-valent vertices. To see this, consider for example a graph which in some neiphborhood $N$ of $\Sigma$ contains a 4 -valent invariant symbol connecting four reps $R_{1}, R_{2}, R_{3}$, and $R_{4}$, as in Fig 2(a). Now consider the decomposition of $R_{1} \otimes R_{2}$ and $R_{3} \otimes R_{4}$ into direct sums of irreducible representations,

$$
R_{1} \otimes R_{2}=\oplus_{n} S_{n} \quad, \quad R_{3} \otimes R_{4}=\oplus_{n} \bar{S}_{n}^{\prime}
$$

It follows that there is an invariant symbol $\left[V_{\left(R_{1} R_{2} \bar{S}_{n}\right)}\right]_{m}^{i j}$ for each $S_{n}$ and another invariant symbol $\left[V_{\left(R_{3} R_{4} S_{n}^{\prime}\right)}\right]^{k l p}$ for each $S_{n}^{\prime}$. (Here $i, j, k, l, m$, and $p$ are matrix indices for the reps $R_{1}, R_{2}, R_{3}, R_{4}, S_{n}$ and $S_{n}^{\prime}$ respectively. Changing the height of an index indicates complex conjugation of the corresponding representation.) Now, it is a group theory fact that the number of linearly independent 4-valent invariant symbols which couple $R_{1} \otimes R_{2} \otimes R_{3} \otimes R_{4}$ is equal to the number of reps which appear in both lists $\left\{S_{n}\right\}$ and $\left\{S_{n}^{\prime}\right\}$. This counting must be done with multiplicity, so that if a particular representation appears $a$ times in $\left\{S_{n}\right\}$ and $b$ times in $\left\{S_{n}^{\prime}\right\}$, then it counts $a b$ times. The corresponding basis of 4 -valent invariant symbols which couple $R_{1}, R_{2}, R_{3}$ and $R_{4}$ are just given by all of the possibilities

$$
\left[V_{(S)}\right]^{i j k l}=\left[V_{\left(R_{1} R_{2} \bar{S}\right)}\right]_{m}^{i j}\left[V_{\left(R_{3} R_{4} S\right)}\right]^{k l m}
$$

So we can expand the 4-valent vertex in Fig. 2(a) in terms of the basis (2.7), and then insert segments in the reps $S$, to separate the trivalent vertices. We can deform the graph, stretching the segments $S$ from infinitesimal to finite size, because of the constraint (2.3) which guarantees that the gauge field is flat everywhere. So we can replace the graph in 
Fig. 2(a) by a sum of corresponding graphs which differ from the original only in that the segments in the neighborhood are reconnected using only 3-valent vertices as in Fig. 2(b). An easy generalization of this argument can be used to also write $n$-valent vertices (for all $n \geq 4$ ) as a sum of graphs which contain only 3 -valent vertices. This is one aspect of the redundancy of the Wilson graphs in Chern-Simons gauge theory, which we hereby take care of simply by resolving to consider from now on only graphs which have at most 3-valent vertices. (In particular, we do not need to consider graphs which have segments which intersect each other in $\Sigma$, since this is just the special case that $R_{3}=\bar{R}_{1}$ and $R_{4}=\bar{R}_{2}$ in the above discussion.) Among the graphs with only 3-valent vertices, there is still some redundancy. An easy way to see this is to note that one could just as easily have employed the argument of the previous paragraph, but with the roles of the $R_{2}$ and $R_{4}$ segments exchanged. Thus we find that the same initial graph in Fig. 2(a) could also have been written as a sum of graphs of the type shown in Fig. 2(c). Comparing Figs. 2(b) and 2(c) shows that there must be a set of redundancy rules for graphs with 3-valent vertices which schematically takes the form indicated in Fig. 3. In order to be concrete, we must first make a specific choice for the normalization of the 3-valent invariant symbols of the group $\mathcal{G}$. It is natural and convenient to do this in such a way that they form an orthonormal basis. For any three representations $R_{1}, R_{2}, R_{3}$ consider the invariant symbols $\left[V_{(n)}\right]^{i_{1} i_{2} i_{3}}$ which couple them. First we would like to specify that the $\left[V_{(n)}\right]^{i_{1} i_{2} i_{3}}$ are independent of the order that we take the representation, so that they are invariant under permutations of the indices. Actually, this convention must be modified only in the special case that the invariant symbol couples two identical reps antisymmetrically [6]. Then a permutation of indices which changes their cyclic order must be accompanied by a minus sign. So we have

$$
\begin{aligned}
{\left[V_{(n)}\right]^{i_{1} i_{2} i_{3}} } & =\left[V_{(n)}\right]^{i_{2} i_{3} i_{1}}=\left[V_{(n)}\right]^{i_{3} i_{1} i_{2}}=(-1)^{V_{(n)}}\left[V_{(n)}\right]^{i_{1} i_{3} i_{2}} \\
& =(-1)^{V_{(n)}}\left[V_{(n)}\right]^{i_{3} i_{2} i_{1}}=(-1)^{V_{(n)}}\left[V_{(n)}\right]^{i_{2} i_{1} i_{3}}
\end{aligned}
$$

where $(-1)^{V_{(n)}}$ is defined to be -1 if any two of $R_{1}, R_{2}$ and $R_{3}$ are identical and coupled antisymmetrically, and is +1 otherwise. Now we require the normalization convention that

$$
\left[V_{(n)}\right]^{i_{1} i_{2} i_{3}}\left[V_{(m)}\right]_{j_{1} i_{3} i_{2}}=\delta_{(n),(m)} \delta_{j_{1}}^{i_{1}}\left(\frac{\operatorname{dim} R_{2} \operatorname{dim} R_{3}}{\operatorname{dim} R_{1}}\right)^{1 / 2}
$$


To see why this normalization is natural, we can contract the remaining $R_{1}$ indices in (2.9) to find the symmetric result

$$
\left[V_{(n)}\right]^{i_{1} i_{2} i_{3}}\left[V_{(m)}\right]_{i_{1} i_{3} i_{2}}=\delta_{(n),(m)}\left(\operatorname{dim} R_{1} \operatorname{dim} R_{2} \operatorname{dim} R_{3}\right)^{1 / 2} .
$$

The result (2.10) is just the classical value of the Wilson graph shown in Fig. 4 which is trivial in the sense that it encloses no topological disturbances and thus has no non-trivial Poisson brackets and so can be set equal to a number. (Note that the indices on $V_{(n)}$ and $V_{(m)}$ must be in the opposite cyclic order in (2.10) to agree with Fig. 4.) Now if we take e.g. $R_{3}=0$ (the trivial rep), then we have $R_{2}=\bar{R}_{1}$ and the graph in Fig. 4 just reduces to a trivial Wilson loop which is equal to the number $\operatorname{dim} R_{1}$, as expected.

We can now proceed to derive a non-trivial redundancy rule for Wilson graphs containing 3-valent vertices. Consider a graph $G_{A}$ which in some neighborhood $N$ of $\Sigma$ contains two line segments in the reps $R_{1}$ and $R_{2}$ of $\mathcal{G}$. By taking the neighborhood $N$ to be small enough, the portions of the Wilson line segments inside it can be set equal to the identity matrices in their respective representations. We can thus write this graph as

$$
G_{A}=\left[g_{A}\right]_{i k}^{j l} \delta_{j}^{i} \delta_{l}^{k}
$$

where $i, j$ are matrix indices for the rep $R_{1}$ and $k, l$ are matrix indices for the rep $R_{2}$, and $\left[g_{A}\right]_{i k}{ }^{j l}$ represents the part of the graph outside of the infinitesimal neighborhood $N$. Now the important feature of $(2.11)$ is that the object $\delta_{j}^{i} \delta_{l}^{k}$ is clearly an invariant symbol of $\mathcal{G}$ which couples the four representations $R_{1}, R_{2}, \bar{R}_{1}$ and $\bar{R}_{2}$. Therefore, it must be possible to expand it in the basis of 4 -valent invariant symbols described by eq. (2.7);

$$
\delta_{j}^{i} \delta_{l}^{k}=\sum_{n, m} b_{(n),(m)}\left[V_{(n)}\right]^{i k p}\left[V_{(m)}\right]_{l j p}
$$

(Here $p$ is a matrix index in the "intermediate" representation $S_{n}=S_{m}$.) One can calculate the coefficients $b_{(n),(m)}$ by multiplying both sides of $(2.12)$ by $\left[V_{(n)}\right]_{i k p^{\prime}}$. With the orthonormality convention (2.9), one obtains;

$$
b_{(n),(m)}=\delta_{(n),(m)}\left(\frac{\operatorname{dim} S_{n}}{\operatorname{dim} R_{1} \operatorname{dim} R_{2}}\right)^{1 / 2}
$$


So we have obtained the result

$$
G_{A}=\left[g_{A}\right]_{i k}{ }^{j l} \sum_{n}\left(\frac{\operatorname{dim} S_{n}}{\operatorname{dim} R_{1} \operatorname{dim} R_{2}}\right)^{1 / 2}\left[V_{(n)}\right]^{i k p}\left[V_{(n)}\right]_{l j p}
$$

Now we can insert segments in the representations $S_{n}$ in between the trivalent vertices as before. We now have obtained the result given in Fig. 5, which says that the original graph containing segments in the reps $R_{1}$ and $R_{2}$ is equal to a sum of graphs which are the same outside the neighborhood, and differ from the original only in the way that the lines are fused inside the neighborhood, as shown.

The redundancy rule of Fig. 5 can be used to express Wilson graphs in terms of related Wilson graphs which have more vertices. There are also redundancy rules which one can apply in certain situations to reduce the number of vertices in a graph, essentially by excising trivial parts of a graph. To understand this, consider the situation in Fig. 6, in which a line in the rep $R$ somewhere in a graph splits at a vertex into two lines $T$ and $S$ which join at a vertex of the same kind into a line $R$ again. The segments $T$ and $S$ do not encircle any topological features of the manifold, and so can be deformed to a point. Because the spatial part of the gauge field is locally flat, we are free to set the lines $S$ and $T$ equal to the identity matrices in their respective representations. Thus from eq. (2.9) we arrive at the result shown in Fig. 6 in which the trivial part of the graph has been removed at the cost of multiplying the graph by a constant factor. More generally, we have the situation depicted in Fig. 7. The blob on the LHS consists of a bunch of segments joined by 3-vertices; let us suppose that it can be deformed to a point without encountering any topological disturbances in the spatial manifold. It car thus be removed from the graph, at the cost of multiplying the graph by a factor of $1 / \operatorname{dim} R$ times the classical value of the graph involving the blob on the RHS, which can be deformed to a point and so is just equal to the appropriate froduct of invariant symbols.

By repeated applications of the two rules in Figs. 5 and 7 , one can find all of the redundancies among classical Wilson graphs containing 3-valent vertices. For example, it is an easy exercise, which we will forego here, to derive the redundancy result indicated schematically in Fig. 3 and compute the coefficients which should appear there. 
We now return to the question of the dynamics of the Wilson graph observables. In cases when two graphs do intersect, we expect that they will have non-vanishing Poisson brackets. Aga.n because of the locality of (2.2), we see that there must be a simple diagrammatic procedure which will allow us to compute the Poisson bracket of any two Wilson graphs. The idea is that since non-zero contributions only come from points of intersection of the two graphs, we can compute the Poisson bracket by adding up the results obtained by applying a rule for breaking the Wilson line segments at each of the intersection points in turn and reconnecting the loose ends. The rule can be derived as follovio. Suppose the graph $G_{A}$ and the graph $G_{B}$ intersect at a point $P$ where $G_{A}$ has a Wilson line segment in the representation $R_{1}$ and $G_{B}$ has a segment in the representation $R_{2}$, as depicted in Fig. 8. Now we can write

$$
\begin{aligned}
& G_{A}=\left(g_{A}\right)_{i}^{j}\left[\delta_{j}^{i}+\int d x^{\mu} A_{\mu}^{a}\left(T_{a}^{(1)}\right)_{j}^{i}\right] \\
& G_{B}=\left(g_{B}\right)_{k}^{l}\left[\delta_{l}^{k}+\int d y^{\nu} A_{\nu}^{b}\left(T_{b}^{(2)}\right)_{l}^{k}\right]
\end{aligned}
$$

where the integrals in (2.15) and (2.16) are each over an infinitesimal segment containing the intersection point $P$, and $T_{a}^{(1)}$ and $T_{a}^{(2)}$ are the matrices for the representations $R_{1}$ and $R_{2}$ respectively. The objects $\left(g_{A}\right)_{i}{ }^{j}$ and $\left(g_{B}\right)_{k}{ }^{\prime}$ represent the portions of the graphs which lic outside the infinitesimal neighborhood containing the intersection. Using (2.2) we compute

$$
\left\{G_{A}, G_{B}\right\}_{P . B .}=\frac{4 \pi}{k}\left(g_{A}\right)_{i}{ }^{j}\left(g_{B}\right)_{k}{ }^{l}\left(T_{a}^{(1)}\right)_{j}{ }^{i}\left(T_{a}^{(2)}\right)_{l}{ }^{k} \int d x^{\mu} d y^{\nu} \epsilon_{\mu \nu} \delta^{(2)}(x-y)
$$

Now the integral in (2.17) is equal to \pm 1 depending on an arbitrary choice of orientation. Further, we note that the object $\left(T_{a}^{(1)}\right)_{j}{ }^{i}\left(T_{a}^{(2)}\right)_{l}{ }_{l}^{k}$ is a 4-valent invariant symbol of $\mathcal{G}$ which couples the representations $R_{1}, R_{2}, \overline{R_{1}}$, and $\overline{R_{2}}$. Therefrre we can expand in a basis and write, just as in (2.12):

$$
\left(T_{a}^{(1)}\right)_{j}{ }^{i}\left(T_{a}^{(2)}\right)_{l}{ }^{k}=\sum_{n, m} c_{(n),(m)}\left[V_{(n)}\right]^{i k p}\left[V_{(m)}\right]_{j l p}
$$

The coefficients $c_{(n),(m)}$ can be computed easily by multiplying both sides of $(2.18)$ by $\left[V_{(n)}\right]_{i k p^{\prime}}$ and using the defining property of the invariant symbols:

$$
\left[V_{(n)}\right]^{i^{\prime} j k}\left(T_{a}^{(1)}\right)_{i^{\prime}}{ }^{i}+\left[V_{(n)}\right]^{i j^{\prime} k}\left(T_{a}^{(2)}\right)_{j^{\prime}}^{j}+\left[V_{(n)}\right]^{i j k^{\prime}}\left(T_{a}^{\left(S_{n}\right)}\right)_{k^{\prime}}{ }^{k}=0
$$


With the orthonormality conditions (2.8) and (2.9) we obtain the result

$$
c_{(n),(m)}=(-1)^{V_{(n)}} \frac{1}{2} \delta_{(n),(m)}\left(C_{R_{1}}+C_{R_{2}}-C_{S_{n}}\right)\left(\frac{\operatorname{dim} S_{n}}{\operatorname{dim} R_{1} \operatorname{dim} R_{2}}\right)^{1 / 2}
$$

where $C_{R}$ denotes the quadratic casimir invariant of the rep $R$. So we have

$$
\begin{aligned}
& \left\{G_{A}, G_{B}\right\}_{P B}= \\
& \quad( \pm 1)(-1)^{V_{(n)} \frac{2 \pi}{k}}\left(g_{A}\right)_{i}{ }^{j}\left(y_{B}\right)_{k}{ }^{l} \sum_{n}\left[V_{(n)}\right]^{i k p}\left[V_{(n)}\right]_{j l p}\left(C_{R_{1}}+C_{R_{2}}-C_{S_{n}}\right)\left(\frac{\operatorname{dim} S_{n}}{\operatorname{dim} R_{1} \operatorname{dim} R_{2}}\right)^{1 / 2}
\end{aligned}
$$

Now we can again employ the trick of deforming the graphs on the RIIS of (2.21), pulling the new 3-valent vertices apart and inserting segments in the representatiuns $S_{n}$. Finally, we resolve the arbitrary choice of orientation sign in (2.21). If the orientation of segments is such that the lines going into the invariant symbols $V_{(n)}$ are in the cyclic order $R_{1}, R_{2}$, $S_{n}$ traveling counterclockwise around the vertex, then we choose the + sign in eq. (2.21); otherwise we choose the minus sign. Thus we have the final result that the contribution to the Poisson bracket of $G_{A}$ and $G_{B}$ due to the intersection point $P$ is given by the sum of graphs in Fig. 9, with the constants $c_{(n)}$ given by

$$
c_{(n)}=(-1)^{V_{(n)}} \frac{2 \pi}{k}\left(C_{R_{1}}+C_{R_{2}}-C_{S_{n}}\right)\left(\frac{\operatorname{dim} S_{n}}{\operatorname{dim} R_{1} \operatorname{dim} R_{2}}\right)^{1 / 2}
$$

and we are instructed to add up all such contributions from each of the intersection points.

We are finally ready to present our alternative definition of canonical classical ChernSimons gauge theory in $2+1$ dimensions. To do this, we first forget about the gauge field $A_{\mu}$ and the action (2.1). Instead, we assert that our theory is a theory of graphs on $\Sigma$ mide out of segments and 3-valent vertices corresponding to representations and invariant syı.nbols of $\mathcal{G}$. Any two graphs which can be smoothly deformed into each other are declared to be equivalent. We do not specify an action principle. Instead, we postulate that the Poisson bracket of any two Wilson graphs is given by the simple graphical rule of Fig. 9 , and that the redundancy rules depicted in Figs. 5 and 7 apply.

It is perhaps not a priori obvious that the postulates of the theory described in the previous paragraph are consistent with each other. For example consider two graphs which 
do not intersect but which can be smoothly deformed in $\Sigma$ and thus redrawn so that they do intersect in two or more points. An important consistency condition then is to check that the contributions to the Poisson bracket from the new intersection points must cancel in the end (since we know from the original non-intersecting presentation of the two graphs that they must have zero Poisson bracket). Another a priori non-trivial consistency check is that the Poisson bracket algebra computed according to (2.21) must obey the Jacobi identity. It is an amusing exercise to show that these conditions are indeed obeyed in various simple situations.

Indeed, one could attempt to derive new theories which have the same qualitative structure as Chern-Simons gauge theory defined in our graphical approach, but which are not necessarily associated with local gauge field theories. That is, one could attempt to define a theory by picking a set of labels for segments and vertices of graphs, and choose a set of rules for computing Poisson brackets and identifying redundancies among different graphs. If one picks such a set of rules completely at random, then it, is no longer clear that the resulting theory will even be internally consistent. The chances for the viability of such a theory seem exceedingly remote, since e.g. the Jacobi identity imposes very non-trivial conditions. It is an interesting open question whether one can define such a theory which is not in some sense equivalent to a Chern-Simon. gauge theory.

\section{Quantum Topological Gauge Theory as a Theory of Graphs}

We can now proceed to quantize our thenry of graphs. In order to be as concrete as possible, we will assume from now on that the spacelike hypersurfaces $\Sigma$ are compact genus $g$ surfaces. First let us consider, in general terms, what it should mean to quantize a theory of graphs, as opposed to a theory of gauge fields. In the usual approach based on gauge fields, in what we might refer to as the "connection representation" of the quantum theory, the wavefunctions $\Psi\left[A_{\mu}\right]$ are functionals of flat $\mathcal{G}$ connections $A_{\mu}$ on $\Sigma$ modulo gauge transformations. (See e.g. [7].) The commutator of two gauge fields is defined by promoting the gauge field to a quantum operator and promoting the canonical Poisson 
bracket algebra to a commutator algebra:

$$
\left[A_{\mu}^{a}(x), A_{\nu}^{b}(y)\right]=\frac{4 \pi i}{k} \epsilon_{\mu \nu} \delta^{a b} \delta^{(2)}(x-y)
$$

Note that the quantity $1 / k$ plays the role of $\hbar$ in the quantum theory. Thus it is a general feature that quantum corrections to the classical physics are given by terms of higher order in $1 / k$. The action of $A_{\mu}$ on the wavefunctions must form a representation of the commutator algebra (3.1). This in turn (at least formally) determines the action of the graph observables on the wavefunctions $\Psi\left[A_{\mu}\right]$.

In our "graph representation" approach, the wavefunctions are functions $\Phi\left[G_{\alpha}\right]$ of a certain subset $\Gamma$ of all possible graphs. The subset $\Gamma$ is a complete set of commuting observables. Then for each graph $G_{\alpha} \in \Gamma$, the wavefunction $\Phi\left[G_{\alpha}\right]$ assigns a particular complex number. (We will be more precise later about exactly which graphs shculd be allowed as arguments of the wavefunctions.) Any state can be expanded in the basis of states $\left|G_{\alpha}\right\rangle$ which hive unit support only on a particular given graph $G_{\alpha}$;

$$
|\Phi\rangle=\sum_{\alpha}\left|G_{\alpha}\right\rangle \Phi\left[G_{\alpha}\right]
$$

The space of wavefunctions needs to be endowed with an inner product, which will be specified by giving the values of the overlaps $\left\langle G_{\alpha} \mid G_{\beta}\right\rangle$ for all states $G_{\alpha}, G_{\beta} \in \Gamma$.

In the graph representation, the graph observables are promoted to the fundamental operators in the theory. The commutator algebra of graphs must be a consistent quantum deformation of the non-canonical Poisson bracket algebra (2.4);

$$
\left[G_{A}, G_{B}\right]=\frac{i}{k} \sum_{C} \hat{F}_{A B}^{C} G_{C}
$$

where the quantum structure constants should be equal to their classical counterparts up to quantum corrections:

$$
\hat{F}_{A B}^{C}=F_{A B}^{C}+\mathrm{O}(1 / k)
$$

This must be accompanied by an appropriate quantum deformation of the redundancy rules;

$$
\sum_{A} \hat{l}_{i}^{A} G_{A}=0
$$


where again the constant $\hat{l}_{i}^{A}$ appearing in (3.5) are the same as their classical counterparts $l_{i}^{A}$ in the large $k$ limit;

$$
\hat{l}_{i}^{A}=l_{i}^{A}+\mathrm{O}(1 / k)
$$

Finding the quantum corrections in (3.4) and (3.6) is not an exercise in arbitrariness, because the Jacobi identity and the internal consistency of (3.3) and (3.5) in practice place very strict conditions on the quantum correction terms. Indeed, for simple choices of gauge group and reps, one can easily construct solutions to (3.3) and (3.5) by working order by order in $(1 / k)$, and demanding that the Jacobi identity of the commutator algebra works at each stage when (3.5) is taken into account. In practice one finds that one must have terms of all orders in $(1 / k)$ in both (3.4) and (3.6). Once a consistent quantum algebra consisting of ( 3.3$)$ and (3.5) has been found, the final step in the quantization procedure is to realize this algebra on the space of states $\Phi\left[G_{\alpha}\right]$. This means that we must give a prescription for computing the action of any graph observable on the basis elements $\left|G_{\alpha}\right\rangle$; so we need to provide a set of structure constants $H_{A \alpha}^{\beta}$ such that

$$
G_{A}\left|G_{\alpha}\right\rangle=H_{A \alpha}^{\beta}\left|G_{\beta}\right\rangle
$$

By comparison of (3.7) with (3.3) and (3.5) we see that we must have

$$
H_{A \gamma}^{\beta} H_{B \alpha}^{\gamma}-H_{B \gamma}^{\beta} H_{A \alpha}^{\gamma}=\frac{i}{k} \hat{F}_{A B}^{C} H_{C \alpha}^{\beta} \quad ; \quad \hat{l}_{i}^{A} H_{A \alpha}^{\beta}=0 \quad \text {. }
$$

The inner product must of course be chosen so that the graph observables are Hermitian operators.

The quantization of a local field theory generally requires the introduction of a regularization scheme. Because of the $\delta$-function occuring in (3.1), the connection representation quantization of topological gauge theory will technically involve divergent expressions reflecting the short distance problems caused by collisions between local operators. In Witten's approach, these problems are avoided by making the formal connection between the three-dimensional quantum field theory and the known results of current algebra in two dimensions. Still, it is worth noting that the problem of providing the theory with a gauge-invariant regulator which rigorously eliminates all the divergences at the level of 
the action (2.1) is a rather thorny one. (The use of e.g. dimensional regularization is at odds with the appearance of the $\epsilon^{\mu \nu \rho}$ in (2.1).) The graph representation of the theory exposes the fact that worries about regularization for topological gauge theory are indeed nothing more than technical details. The divergences in the connection representation are just fakes which can be blamed on the fake degrees of freedorn in $A_{\mu}^{a}$. The Poisson bracket algebra (2.2) and commutator algebra (3.3) contain no infinities and no $\delta$-functions; the structure constants $\hat{F}_{A B}^{C}$ and $\hat{l}_{i}^{A}$ are just finite numbers. So an advantage of the graph representation is that it obviates the use of formal arguments to eliminate the divergences which are introduced along with the fake degrees of freedom in the gauge field.

One way of regularizing graph observables in the connection representation is to introduce a "framing" for graphs. This is essentially a thickening of the segments of the graph into ribbons, so as to prevent short distance divergences from $A_{\mu}^{a}$ 's hitting each other. There are many ways to thicken a graph. So the choice of framing convention introduces an ambiguity inherent in defining Wilson graphs as quantum observables. This ambiguity manifests itself as terms of higher order in $1 / k$ in various formulas. In the graph representation, these ambiguities also arise, but in a rather different way. The graphs are no longer defined in terms of gauge fields with divergent correlators, so there is no reason to thicken the graphs. However, there are different ways to consistently pick the quantum corrections in (3.4) and (3.6); these just correspond to the framing ambiguities in the connection representation.

Now, the problem of quantizing the classical theory defined by (2.4) and (2.5) is certainly a. well-defined and self-contained one, which can in principle be solved without resorting to outside tricks. However, there is no obvious simple prescription for systematically carrying out this quantization other than to simply guess. One could of course work out the quantization by working constructively and order-by-order in $\hbar \sim 1 / k$ and indeed we have done this in some simple cases, but this can be messy in general. Fortunately, we can use the results of [3-5] (which enable us to calculate the expectation value of any collection of Wilson graphs), as a shortcut to help us guess. This is motivated by an obvious qualitative similarity between our redundancy rules of Figs. 5 and 7 and Poisson 
bracket rule Fig. 9 on the one hand, and the skein relations described by Witten in [4] on the other. In Witten's approach, one can calculate the expectation value of an arbitrary collection of knotted graphs using skein relations and factorization rules. Skein relations are linear identities which hold between expectation values of Wilson graphs which are the same outside of some 3-ball $B$ and which are different inside of $B$. Suppose that four lines, in the reps $R_{1}, R_{2}, \bar{R}_{1}$ and $\bar{R}_{2}$, enter $B$ and are connected there by 3 -vertices in various ways. A skein relation says that the total of some number of such expectation values, with computable coefficients, must vanish. The skein relations are pictured projected to a plane, with the three dimensional information captured by indicating which segments overlap others in the projection.

Witten introduces a particularly convenient framing convention called "vertical framing". This involves imagining flattening a graph by projecting it into a horizontal plane and then thickening each segment of the graph in the direction perpendicular to that plane. A slight variant of this convention, which we might call "time-like framing" is most naturally applicable to the canonical approach. If the spacetime manifold is $\Sigma \otimes$ time, a graph lying in $\Sigma$ is thickened in the direction of increasing time. This way, in any neighborhood $N$ of $\Sigma$ which is devoid of topological disturbances, this framing convention is the same as vertical framing, with the vertical direction being the time direction. In the graph representation we never actually thicken our graphs, but we can naturally pick the ambiguities in (3.4) and (3.6) to correspond to this convention. The classical redundancy relations and Poisson brackets of our canonical graph observables can then be viewed loosely as corresponding to the classical $O\left((1 / k)^{0}\right)$ and leading quantum part $O\left((1 / k)^{1}\right)$ of the skein relations, respectively. This is essentially the Correspondence Principle at work.

Indeed, with the correct interpretation, we can simply appropriate the picture corresponding to one of the skein relations (with vertical framing) given by Witten and adopt it as the quantum deformation of the classical redundancy relation. The relevant skein relation is exactly the same picture shown earlier in Fig. 5 but now with the coefficients 
given by

$$
b_{(n),(m)}=\delta_{(n),(m)} \frac{S_{0, S_{n}} S_{0,0}}{S_{0, R_{1}} S_{0, R_{2}}}
$$

where the $S_{i, j}$ are the components of the matrix which represents the $\tau \rightarrow-1 / \tau$ modular transformations of the characters in the corresponding 2-d current algebra. The only important thing we need to know about this matrix is that

$$
S_{0, R} / S_{0,0}=\operatorname{dim} R+\mathrm{O}\left(1 / k^{2}\right) .
$$

So in the classical limit $k \rightarrow \infty$ the vertically framed skein relation of Fig. 5 with (3.9) corresponds exactly to our redundancy rule given previously. Now it is perhaps not immediately obvious that the skein relation, which tells us about expectation values of operators, can be adopted as a redundancy relation, which is supposed to equate linear combinations of the operators themselves. The reason it works is that the skein relations hold regardless of whatever else is going on elsewhere in the 3 -manifold $\mathcal{M}$. So we probationarily adopt Fig. 5 with (3.9) as the exact quantum redundancy relation, contingent on our success in finding the rest of the quantization procedure.

Similarly, the quantum deformation of the redundancy rule of Fig. 7 is exactly the same as Fig. 7, except that the factor of $1 / \operatorname{dim} R$ is replaced by $S_{0,0} / S_{0, R}$ and the classical graph with the blob in it is replaced by its covariant expectation value on the 3 -sphere calculated according to the rules of [3-5]. Once again in the classical limit of large $k$, we can see from (3.10) that our quantum redundancy rule just goes over into the classical redundancy relation, as required.

We can also find the quantum deformation of the Poisson bracket rule into a commutator algebra by using appropriate skein relations. To make this work, we want to make a formal connection between certain expectation values of (unknotted) graphs in Witten's covariant approach and matrix elements of spacelike graph observables in the canonical approach. In the canonical graph representation approach, there is a natural vacuum state |0) which has support only on the trivial graph. Now we can imagine acting on this state successively with a string of graph observables which include two graphs $G_{A}$ and $G_{B}$ whose commutator we are interested in finding, and then taking the inner product of this state 
with the vacuum state $\langle 0|$. Then subtract the same thing but with the roles of the graphs $G_{A}$ and $G_{B}$ reversed. So the object we are interested in is;

$$
\left\langle 0\left|\ldots G_{X}\left[G_{A}, G_{B}\right] G_{Y} \ldots\right| 0\right\rangle=\left\langle 0\left|\ldots G_{X} G_{A} G_{B} G_{Y} \ldots\right| 0\right\rangle-\left\langle 0\left|\ldots G_{X} G_{B} G_{A} G_{Y} \ldots\right| 0\right\rangle
$$

Now each of the operators in this formula lives on the 2 -surface $\Sigma$. Introduce a time coordinate $t$ whose only role is to measure the order that the graphs are applied to the ket vacuum $|0\rangle$. Thus in the RHS of (3.11), the order of graphs in increasing time is $\ldots G_{Y} G_{B} G_{A} G_{X} \ldots$ in the first term, and $\ldots G_{Y} G_{A} G_{B} G_{X} \ldots$ in the second term. This time-ordering allows us to express (3.11) in the formal three-dimensional covariant form

$$
\left\langle 0\left|\ldots G_{X}\left[G_{A}, G_{B}\right] G_{Y} \ldots\right| 0\right\rangle=\int\left[\mathrm{d} A_{\mu}^{\prime}\right] e^{i S_{C S}}\left(\ldots G_{X} G_{A} G_{B} G_{Y} \ldots-\ldots G_{X} G_{B} G_{A} G_{Y} \ldots\right)
$$

where now the three-dimensional arrangement of graphs on the RHS of (3.12) is understood to be just as indicated by our time ordering. But now the expectation value on the RHS of (3.12) can be partially evaluated by repeated use of Witten's skein relations and factorization rules. The point is that the RHS reduces to the difference of two expectation values of graphs built out of $\ldots G_{X} G_{A} G_{B} G_{Y} \ldots$ which only differ at points where the graphs $G_{A}$ and $G_{B}$ overlap when projected through the time direction onto the 2-manifold $\Sigma$ (with segments at later times lying on top of segments at earlier times). Consider a small neighborhood where graph $G_{A}$ has a segment in the rep $R_{1}$ and $G_{B}$ has a segment in the rep $R_{2}$ as depicted on the LHS of Fig. 10. At the overlap point, the two graphs only differ in which of the segments is on top of the other. Now we can find the appropriate skein relations for these two configurations in ref. [8]; they are shown in Figs. 10 and 11, and the coefficients $\phi_{(n)}$ are given by

$$
\phi_{(n)}=(-1)^{V_{(n)}} \exp \left[i \pi\left(C_{R_{1}}+C_{R_{2}}-C_{S_{n}}\right) /\left(k+C_{A d j}\right)\right] \frac{S_{0, S_{n}} S_{0,0}}{S_{0, R_{1}} S_{0, R_{2}}}
$$

Note that when the order of overlaps are reversed, the coefficients are just complex conjugated. By applying these skein relations to all of the overlap points of $G_{A}$ and $G_{B}$ on the 
RHS of (3.12), one reduces it to the form

$$
\sum_{C} \hat{F}_{A B}^{C} \int\left[\mathrm{d} A_{\mu}^{\prime}\right] e^{i S_{C S}}\left(\ldots G_{X} G_{C} G_{Y} \ldots\right)
$$

where the graphs $G_{C}$ have no ambiguous self-intersections when projected along the time direction onto $\Sigma$, and the structure constants $\hat{F}_{A B}^{C}$ are determined directly from the repeated use of the skein relations in Figs. 10 and 11. The key point is that this procedure is completely independent of the other graphs which are present. This means that we can set the commutator of the graphs $G_{A}$ and $G_{B}$ equal to $\hat{F}_{A B}^{C} G_{C}$ (as computed from the skein relations) as an operator equation, and this answers the question of how to determine the quantum commutator coefficients. The important thing is that in the classical limit of large $k$, we recover the Poisson bracket rule given in section 2. It is easy to see that this is indeed the case, because in the large $k$ limit each intersection point will just make an independent additive contribution given by the $k \rightarrow \infty$ limit of the difference of Figs. 10 and 11 , and because

$$
\lim _{k \rightarrow \infty}\left(\phi_{(n)}-\phi_{(n)}^{*}\right)=i c_{(n)}
$$

Now that we have made a proposal for the quantum redundanc $c^{\prime}$ and commutator rules, it remains to show that we can realize this algebra on a space of states $\left|G_{\alpha}\right\rangle\left(G_{1 x} \in \Gamma\right)$ with a suitable inner product. The subset of graphs $\Gamma$ ought to be a complete set of commuting observables. There is no canonical way to pick $\Gamma$, but here is one way. Consider the genus $g$ spatial hypersurface $\Sigma$ to be imbedded in $R^{3}$, and fill in the interior of $\Sigma$, to form a solid handlebody $\tilde{\Sigma}$. Then one chooses a "pre-graph" lying entirely inside $\widetilde{\Sigma}$ and of which $\tilde{\Sigma}$ is a neighborhood. The pre-graph is constructed out of segments connected by 3 -vertices, but the segments are not yet labeled by representations of $\mathcal{G}$ and the vertices are not yet labeled by invariant symbols of $\mathcal{G}$. The pre-graph must be chosen so that no part of it is contractible in $\widetilde{\Sigma}$, and so that any non-contractible loop on the surface $\Sigma$ which is contractible in $\tilde{\Sigma}$ must encircle a segment of the pre-graph. We have illustrated two possible choices for the pre-graph for genus two, in Fig. 12. The basis for the set of graphs to use for $\Gamma$ is just given by labelings of the segments and vertices of one of the pre-graphs. One would not want to put graphs from both pre-graphs of Fig. 12 into $\Gamma$, as this would lead 
to an overcounting of states, and a reducible representation of the quantum commutator algebra. This is because the labelings of each valid pre-graph separately forms a basis for the space of states. The elements of these two bases can be related e.g. by the redundancy rules. The representation labels for the segments are chosen from a subset of all possible reps of $\mathcal{G}$. Naively, one should allow the segments to be labelled by any representations of $\mathcal{G}$, but we will see later that this is not necessarily quite the correct thing to do.

The inner product on the space of states is just the obvious one. In terms of the basis elements $\left|G_{\alpha}\right\rangle\left(G_{\alpha} \in \Gamma\right)$, we have

$$
\left\langle G_{\alpha} \mid G_{\beta}\right\rangle=\delta_{\alpha \beta}
$$

That is, if two graphs are exactly identical (in every label), then the overlap of the corresponding basis elernents $\left\langle G_{\alpha} \mid G_{\beta}\right\rangle$ is unity; otherwise it is zero. (Witten defines an inner. product in [4] which differs from this one by an overall genus-dependent normalization factor. In our situation with $\mathcal{M}=\Sigma \times$ time, this normalization factor is of no significance. Of course, if we were going to consider topology-changing situations, we could not be so cavalier about the overall normalization of eq. (3.16).) Thus we have the completeness relation

$$
1=\sum_{G_{\alpha} \in \Gamma}\left|G_{\alpha}\right\rangle\left\langle G_{\alpha}\right|
$$

Now by writing

$$
G_{A}\left|G_{\alpha}\right\rangle=\sum_{\beta}\left|G_{\beta}\right\rangle\left\langle G_{\beta}\left|G_{A}\right| G_{\alpha}\right\rangle
$$

the problem of realizing the commutator algebra on the space of states is reduced to that of providing a prescription for computing the canonical matrix elements $H_{A \alpha}^{\beta}=\left\langle G_{\beta}\left|G_{A}\right| G_{\alpha}\right\rangle$. We do this by relating it in a formal way to a corresponding covariant expectation value which can be computed by the methods of ref. [4]. We can think of $\left|G_{\alpha}\right\rangle$ as being the state corresponding to an empty handlebody $\widetilde{\Sigma}$ with an insertion of the graph operator $G_{\alpha}$ inside it. The graph observable $G_{A}$ acts on the surface $\Sigma$ which is the boundary of $\widetilde{\Sigma}$. Finally $\left\langle G_{\beta}\right|$ corresponds to an insertion of the graph $G_{\beta}$ inside a second copy of $\widetilde{\Sigma}$ which 
is glued to the first one on the common boundary $\Sigma$. Then we have, as pictured in Fig. 13

$$
\left\langle G_{\beta}\left|G_{A}\right| G_{\alpha}\right\rangle=\left\langle G_{\beta} G_{A} G_{\alpha}\right\rangle_{\widetilde{\Sigma}_{\circ} \widetilde{\Sigma}} /\langle 1\rangle_{\tilde{\Sigma} \circ \widetilde{\Sigma}}
$$

whic! relates the canonical matrix element to a covariant expectation value on the compact 3-manifuld gotten by gluing two copies of the handlebody together. The RHS of eq. (3.19) (Fig. 13) can be computed by using skein relations and factorizing on 2-spheres with the techniques of ref. [4]. The factor of $1 /\langle 1\rangle_{\widetilde{\Sigma}} \circ \widetilde{\Sigma}$ is needed to achieve the correct normalization to agree with (3.16) when we take $G_{A}=1$ (the trivial graph). Eq. (3.19) thus determines the action of the graph observables on an arbitrary basis element. Using the skein relations, it is not too hard to show that the basis elements $\left|G_{\alpha}\right\rangle$ are eigenstates of those Wilson loops on $\Sigma$ which are contractible in the solid handlebody $\widetilde{\Sigma}$ which we used to construct the pre-graph. These Wilson loops can be thought of as measuring the "flux" through the corresponding part of $\Sigma$ in the state $\left|G_{\alpha}\right\rangle$, while the other Wilson graphs which are non-contractible in $\widetilde{\Sigma}$ create and destroy this flux.

Now it is clear that we have successfully conspired to realize the commutator algebra in a way consistent with the redundancy relations, based on the previously known consistency of the skein relations of ref. [4]. For example, consider the action of the quantum redundancy relation on an arbitrary basis element;

$$
\left(\sum_{A} \hat{l}_{i}^{A} G_{A}\right)\left|G_{\alpha}\right\rangle=\sum_{\beta}\left|G_{\beta}\right\rangle\left(\sum_{A} \hat{l}_{i}^{A}\left\langle G_{\beta} G_{A} G_{\alpha}\right\rangle_{\widetilde{\Sigma} \circ \widetilde{\Sigma}}\right) /\langle 1\rangle_{\widetilde{\Sigma}_{\circ} \widetilde{\Sigma}} .
$$

Since we just chose the redunclancy relation coefficients to correspond to the skein relations which are used to compute $\left\langle G_{\beta} G_{A} G_{\alpha}\right\rangle_{\widetilde{\Sigma} \circ \widetilde{\Sigma}}$ it follows that the RHS of (3.20) must vanish as required. By analogous arguments one finds that the commutator algebra is consistently realized by (3.18) and (3.19) as well. Also, the hermiticity of the graph observables with respect to the inner product (3.16) is guaranteed by the fact that in the skein relations, reversing the sense of the overlaps (from over to under and vice versa) just complex conjugates all of the coefficients which appear in them, so that from (3.19) we have immediately

$$
\left\langle G_{\beta}\left|G_{A}\right| G_{\alpha}\right\rangle=\left\langle G_{\alpha}\left|G_{A}\right| G_{\beta}\right\rangle^{*} \quad
$$


At this point we could claim to be finished with the program of exhibiting the quantization of the classical graph theory, except that there are several apparent discrepancies remaining between our results and the standard ones given for quantum Chern-Simons gauge theory defined from the connection approach! The flrst essentially concerns the allowed values for $\hbar$ in the quantum theory. As we mentioned earlier, the coupling constant $k$ which appears in front of the action must be an integer. In the connection representation, this must be so in order that the functional integral weighted by $\exp \left(i S_{C S}\right)$ is invariant under largc gauge transformations. However, in the graph representation approach, where there is no such thing as gauge invariance, we have not yet seen any reason why $k$ could not take on any value whatsoever. A closely related question involves the celebrated "renormalization" $k \rightarrow k+C_{A d j}$ which occurs for example in (3.13). By using the skein relations, we guaranteed that this renormalization would occur automatically, because the skein relations always depend on $k$ through rational powers of the phase

$$
\exp 2 \pi i /\left(k+C_{A d j}\right)
$$

However, within the context of the quantization of the classical theory of graphs, one could imagine replacing the expression $k+C_{A d j}$ everywhere it appears according to

$$
k+C_{A d j} \rightarrow \hat{k}=k+c(k)
$$

where $c(k)$ is any function of $k$ which approaches a constant as $k \rightarrow \infty$. According to our quantization procedure, this would be perfectly valid since in the large $k$ limit the commutator algebra and redundancy relations would still approach the correct classical limits.

It turns out that both of these mysteries of the graph representation are related to the dimensionality of the quantum Hilbert space. The point is that we are indeed free to choose $c(k)$ to be anything we want in (3.22), but if we want to have a finite dimensional Hilbert space, then $\hat{k}=k+c(k)$ must be an integer. Naively, the Hilbert space spanned by the basis vectors $\left|G_{\alpha}\right\rangle$ is infinite dimensional because each of the segments of the pre-graph can be labelled by arbitrary reps of $\mathcal{G}$. But in the special case that $\hat{k}=k+c\left(k_{i}\right)$ is an 
integer, the Hilbert space can be consistently truncated to a finite dimensional Hilbert space, essentially because $\exp (2 \pi i / \hat{k})$ is a root of unity when $\hat{k}$ is an integer. In this case, it turns out to be consistent to take as a basis for $\Gamma$ only those graphs whose segments are labelled by a a finite number of special reps of $\mathcal{G}$. These special reps are of course the integrable highest weight reps of the affine Lie algebra at level $k^{\prime}=\hat{k}^{\prime}-C_{A d j}$.

Let us see how this works for the easy special case of $\mathcal{G}=S U(2)$ and $\Sigma=$ torus. Then $\widetilde{\Sigma}$ is just a solid doughnut, and pre-graph for this situation is just a circle around the $b$-cycle which is non-contractible inside the doughnut. (Thus in this situation the pregraph does not need 3-vertices.) The basis elements for the Hilbert space are labeled by Wilson loops in the spin $j$ rep of $S U(2) ;\left|W_{j}(b)\right\rangle$, or, more succinctly, $|j\rangle$. The simplest graph observables in the theory are then given by Wilson loops which wind once around the $a$ and $b$-cycles in various reps. The Wilson loops which wind once around the $a$-cycle all commute with each other, and likewise the loops which wind once around the b-cycle all commute with each other. The basis elements $|j\rangle$ are eigenstates of the $a$-cycle loops $W_{a}(l)$, and the $b$-cycle loops $W_{b}(l)$ act to create and destroy the flux measured by the $a$-cycle loops. By using the $\mathrm{s}$ kein relations as described above, one can easily compute the action of these loop observables on the basis elements;

$$
\begin{aligned}
& W_{a}(l)|j\rangle=\frac{\sin (\pi(2 j+1)(2 l+1) / \hat{k})}{\sin (\pi(2 j+1) / \hat{k})}|j\rangle \\
& W_{b}(l)|j\rangle=\sum_{p=|j-l|}^{j+l}|p\rangle .
\end{aligned}
$$

In equations (3.23) and (3.24) we have taken the liberty of replacing the quantity $k+C_{A d j}$, which would follow from slavish adherence to the skein relations, by the more general possibility $\hat{k}$ which is supposed to be anything which goes over into $k$ in the classical limit. Now when $\hat{k}$ is an integer, something special happens. In that case we can make an identification of states and operators labeled by different reps, thus simultaneously truncating the Hilbert space and observable algebra in a consistent way. Specifically, for 
every positive integer $m$ we make the identiflcations

$$
\begin{aligned}
& |(m \hat{k}-1) / 2\rangle \rightarrow 0 \\
& |(m \hat{k}+n) / 2\rangle \rightarrow(-1)^{m}|n / 2\rangle \quad n=0,1,2, \ldots, \hat{k}-2
\end{aligned}
$$

for the basis elements and similarly

$$
\begin{aligned}
& W_{(m \hat{k}-1) / 2}(C) \longrightarrow 0 \\
& W_{(m \hat{k}+n) / 2}(C) \longrightarrow(-1)^{m} W_{n / 2}(C) \quad n=0,1,2, \ldots, \hat{k}-2
\end{aligned}
$$

for every Wilson loop observable around a contour $C$. These truncations are clearly respected by (3.23) and (3.24). Thus, for example, in the truncated theory one finds that $(3.24)$ becomes

$$
W_{b}(l)|j\rangle=\sum_{p} N_{l j p}|p\rangle
$$

where $N_{l j p}$ is exactly the Verlinde coupling [9]. More generally, one truncates the theory by setting to zoro all graph states and observables which contain a rep of $\operatorname{spin}(m \hat{k}-1) / 2$, and replacing each occurence of a rep of spin $(m \hat{k}+n) / 2$ for $n=0,1,2, \ldots \hat{k}-2$ with a rep of spin $n / 2$, multiplying the whole graph by $(-1)^{m}$. Each trivalent vertex is of course also replaced with the appropriate colleague according to the replacements of the segments which run into it. (There is a tricky minus sign lurking here, because in some cases the truncations of a graph will cause a vertex to be replaced by one which couples two identical reps antisymmetrically. In that case, one must be careful to choose the correct sign according to the cyclic order of indices.) Using the skein relations one computes;

$$
\sum_{m=|j-l|}^{\left[W_{b}(j), W_{a}(l)\right]=}(-1)^{V_{(j / m)}} G_{j, l, m} \frac{\sin (\pi / \hat{k}) \sin (\pi(2 m+1) / \hat{k}) \sin (\pi[j(j+1)+l(l+1)-m(m+1)] / \hat{k})}{\sin (\pi(2 j+1) / \hat{k}) \sin (\pi(2 l+1) / \hat{k})}
$$
where $G_{j, l, m}$ is the graph pictured in Fig. 14. It is not hard to show that (3.28) respects the indicated truncation of the theory when $\hat{k}$ is an integer. By using the sliein relations, one can show that the commutator of any two graphs of the form in Fig. 15 is always a linear combination of other graphs of this form. Even better, one can show that every 
Wilson graph on the torus can be written as a linear combination of graphs of this same type. This result generalizes in a fairly obvious way to arbitrary genus $g$ surfaces and arbitrary gauge groups $\mathcal{G}$.

For any compact Lie group $\mathcal{G}$, one can make the quantum Hilbert space finite dimensional by truncating in a manner similar to $(3.25)$ and $(3.26)$ when $\hat{k}$ in analogous formulas is an integer. We emphasize that choosing $\hat{k}$ to be an integer, and in particular choosing it to equal $k+C_{A d j}$, does not follow from anything in our definition of the classical theory of graphs. Rather, these are choices made in the quantization process depending on what one wants the dimension of the quantum Hilbert space to be. In this way, the quantization process for the non-canonical Poisson bracket algebra necessarily introduces information present neither in the classical theory of graphs nor in the Correspondence Principle between classical and quantum theories. The renormalization of the classical parameter $k$ to its quantum value, and the very structure of the quantum Hilbert space depend on arbitrary choices made in the quantization procedure in the graph representation approach.

If one insists on having $\hat{k}$ be a non-integer, then the Hilbert space is necessarily infinitedimensional, but nothing else pathological occurs in the graph representation approach,

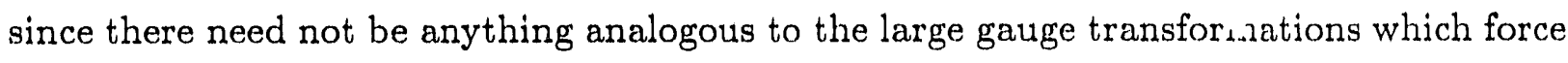
$\hat{k}$ to be an integer in the connection representation approach. Indeed, one may recall from the theory of knot and link polynomials that there is no necessary restriction of the coefficient appearing in the skein relations analogous to the $\hat{k}=$ integer restriction. So the infinite dimensional Hilbert space theories arrived at for $\hat{k} \neq$ integer are the canonical realizations of the field theories which correspond to these more general knot, link, and graph invariants.

So far, we have concentrated on the case that $\mathcal{G}$ is a compact Lie group. Of course there are other interesting possibilities for topological gauge theories in $2+1$ dimensions, which correspond to theories of graphs which do not always quite fit precisely into our discussion here but have the same general character. For example, if we choose $\mathcal{G}$ to be the Poincare group in $2+1$ dimensions, also known as $\operatorname{ISO}(2,1)$, then the resulting theory is equivalent 
to pure Einstein gravity [2]. Gravity in $2+1$ dimensions has long been touted [10-17] as an educational toy model for those who aspire to quantize gravity in the real world. (There are some similarities between the approach of $[18,19]$ based on Ashtekar's new variables [20] for canonical gravity in $3+1$ dimensions and the loop-space approach based on the first order formulation in $2+1$ dimensions.) The role of the Wilson loop observables in $2+1$ gravity and the subsequent loop-space quantization have been discussed in $[21,22,23]$. There are obvious similarities between the loop-space quantization of $2+1$ gravity and the compact $\mathcal{G}$ case we have discussed here, but there are also some important differences. These occur because the $2+1$ gravity case is in some ways simpler due to the structure of the group $I S O(2,1)$, which is the total space of the cotangent bundle of the Lie group $S O(2,1)$. The gauge connection naturally splits into two parts which are interpreted as the spin connection and triad, with canonical Poisson brackets

$$
\left\{e_{\mu}^{a}, \omega_{\nu}^{b}\right\}_{P . B .}=\epsilon_{\mu \nu} \eta^{a b} \delta^{(2)}(x-y) ; \quad\left\{\omega_{\mu}^{a}, \omega_{\nu}^{b}\right\}_{P . B .}=0 ; \quad\left\{e_{\mu}^{a}, e_{\nu}^{b}\right\}_{P, B .}=0
$$

The Wilson loops split naturally into two classes. The first class of loops consist of the "configuration variables" on the reduced phase space (i.e. the remaining phase space after the gauge dependence has been completely eliminated). It consists of Wilson loops which contain only the spin connection $S O(2,1)$ part of the full $I S O(2,1)$ connection, and which do not depend on the triad part at all. The second class of loops are only linear in the triad part of the $\operatorname{ISO}(2,1)$ connection. Because of this splitting of the phase space, it is natural in the quantum theory to make the states in the Hilbert space be functionals of only the first class of loops, and to take the natural vacuum state (which is the basis element corresponding to the trivial loop) to be annihilated by all of the loops in the second class.

To see how this arises, recall the construction of representations of $\operatorname{ISO}(2,1)[22] .(A$ very similar procedure will work for any group which is the total space of the cotangent bundle of another Lie group.) The ISO $(2,1)$ Lie algebra is generated by matrices $J_{a}$ and $P_{b}$ satisfying

$$
\left[J_{a}, J_{b}\right]=\epsilon_{a b c} J^{c} \quad ; \quad\left[J_{a}, P_{b}\right]=\epsilon_{a b c} P^{c} \quad ; \quad\left[P_{a}, P_{b}\right]=0
$$


First, we note that it is easy to find matrix representations of the $S O(2,1)$ subalgebra generated by the $J_{a}$; these are given by setting the $J_{a}$ equal to the usual spin $j$ reps of $\mathrm{SU}(2)$ with some strategic factor of $i$ inserted. To complete this first representation we just set the $P_{u}$ equal to zero identically. This representation of $I S O(2,1)$ yields the first class of Wilson loops mentioned above.

The second representation of (3.30) is obtained by using the same $J_{a}$ 's as in the first representation but now taking $P_{a}=\theta J_{a}$ where $\theta$ is an infinitesimal parameter which is formally taken to satisfy $\theta^{2}=0$. Now we can compute Wilson loops in both of these representations at the same time, just by working in the second representation and setting $\theta=0$ if the first representation is desired. So we take

$$
W_{j, \theta}(L)=\operatorname{Tr} P \exp \oint_{L} d x^{\mu}\left[\omega_{\mu}^{a}+\theta e_{\mu}^{a}\right] J_{a}
$$

The $\theta$-independent part of this expression gives the Wilson loops of the first class, and the part linear in $\theta$ yield the Wilson loops of the second class. Obviously the second class Wilson loops are just the trace of the path-ordered exponential of the spin connection with an insertion of the triad at the basepoint, with the position of the basepoint integrated over. Similarly, one has two classes of Wilson graphs. The first class of graphs just consists of path-ordered exponentials of the spin connection tied together with invariant symbols of $S O(2,1)$. The second class of graphs are again linear in the triad, and can be obtained by putting each segment of the graph in the second representation (using the same $\theta$ for all segments) and then taking the $\theta$ part of the toral result. This is the same as integrating over all possible ways of inserting a single triad in the graph. From the canonical Poisson brackets (3.29) one finds that all of the Wilson graphs of the first class have vanishing Poisson bracket with each other, that the Poisson bracket of a first class graph and a second class graph is always a sum of first class graphs, and that the Poisson bracket of two second class graphs is always a sum of second class graphs.

The construction of the quantum theory of the Poisson bracket algebra is much easier than in the case of compact $\mathcal{G}$, essentially because the quantum correction terms are mush milder. This is because each graph contains at most one triad, and these are used up when 
one takes commutators. So the commutator algebra is essentially the same as the classical Poisson bracket algebra, instead of picking up corrections at each order in $\hbar=1 / k$ as in the compact $\mathcal{G}$ case. Following the case of compact $\mathcal{G}$, we can construct the basis elements by first constructing a solid handlebody $\widetilde{\Sigma}$. The crucial difference is that we should cut out a "core" from the handlebody, which represents the boundary condition that any graph containing a triad should annihilate the vacuum associated with the trivial graph. The result is that the handlebody in the compact $\mathcal{G}$ case is just replaced by $\Sigma$ itself in the gravity case. Then the basis elements are obtained by acting on this vacuum by Wilson graphs of the first class. There are different ways to choose the graphs which label the states in the theory. The way most similar to our discussion for compact $\mathcal{G}$ would be to allow first class graphs with representations of arbitrary spin, connected by tri-valent vertices and with no segments intersecting. It is actually possible to carry this out. However, it is somewhat simpler to just use loops of the first class, only in the fundamental (spin $1 / 2$ ) representation, which may wind around handles in $\Sigma$ an arbitrary number of times (and thus may intersect themselves in $\Sigma$ ). Because this approach is simpler, we will resist the temptation to discuss here the method which uses tri-valent vertices. If one sticks to just the spin- $1 / 2$ representations, then the loops of the first and second class completely parametrize the physical phase space, and the Poisson bracket of any two Wilson loops is a sum of other Wilson loops. The Poisson bracket algebra and classical redundancy rules for this theory of loops have already been discussed in $[21,22,23]$. One can go ahead and carry out the graph representation quantization using only loops, and this is what has been done in [21]. The states in this approach are functionals on the space of loops of the first class. On a surface $\Sigma_{g}$ of genus $g$, one takes the basis elements to be wavefunctions which have support on any loop, rather than just those loops which are non-contractible in a handlebody associated with $\Sigma_{g}$, as we did for the case of compact $\mathcal{G}$.

\section{Conclusion}

In this paper we have shown how one can regard topologicai gauge theory in three 
dimensions as a theory of non-local graph observables governed by a local dynamics. This shows that it is possible, in a particularly simple case, that a theory of geometry can be defined without using connections, metrics, or other gauge-dependent quantities. One advantage of formulating things in terms of observables is of course that only physical degrees of freedom are present from the start, and one does not have to struggle to separate the physics from the gauge. Another interesting feature is that there are no infinities encountered in the quantisation procedure; these are all eliminated because the non-locality of the graph variables "smears out" the delta-functions found in the classical dynamics of the connection variables. Of course, it is much more difficult to carry out such a program when the gauge theory is non-topological. When there are local degrees of freedom present, the graph observables cannot be deformed at will, so that there are uncountably infinitely many more of them. Another enormous qualitative difference is that the observables will typically contain insertions of the momenta conjugate to the connections inserted at points in the graph, because the momentum conjugate to a connection will typically transform homogeneously, unlike the connection itself. The idea that gravity in $3+1$ dimensions can be defined in terms of an algebra of loop variables has been developed extensively by Rovelli and Smolin in [19] using the new canonical variables proposed by Ashtekar [20]. There are of course still tremendous obstacles remaining in the program of quantizing gravity in $3+1$ dimensions, but it is at least interesting that the classical theory of Einstein's gravity can be defined entirely in terms of the loop variables. One might go so far as to speculate that the fundamental theory including both gravity and quantum mechanics should be formulated only in terms of non-local observables, and that a more conventional formulation involving metrics and connections may only arise in an appropriate classical low energy limit.

I am grateful to Richard Woodard for helpful comments. This work was supported in part by the Institute for Fundamental Theory and by DOE contract DE-FG05-86-ER40272. 


\section{References}

1. S. Mandelstam, Ann. Phys. NY 19, 1, (1962); Ann. Phys. NY 19, 25, (1962).

2. E. Witten, Nucl. Phys. B311, 46, (1988).

3. E. Witter, Commun. Math. Phys. 121, 351, (1989).

4. E. Witten, Nucl. Phys. B322, 629, (1989).

5. E. Witten, Nucl. Phys. B330, 285, (1990).

6. G. Moore and N. Seiberg Phys. Lett. B212,451, (1988); Comm. Math. Phys. 123, 177, (1989); Nucl. Phys. B313, 16, (1989).

7. S. Elitzur, G. Moore, A. Schwimmer and N. Seiberg Nucl. Phys. B326, 108, (1989).

8. S. P. Martin, Nucl. Phys. B338, 244, (1990).

9. E. Verlinde, Nucl. Phys. B300, 360, (1988).

10. S. Deser, R. Jackiw, and G. 't Hooft, Ann. Phys. NY 152, 220, (1984).

11. S. Deser and R. Jackiw, Ann. Phys. NY 153, 405, (1984).

12. J. R. Gott and M. Alpert, Gen. Rel. Grav. 16, 243, (1984).

13. S. Giddings, J. Abbott and K. Kuchar, Gen. Rel. Grav. 16, 751, (1984).

14. E. Martinec, Phys. Rev. D30, 1198, (1984).

15. T. Banks, W. Fischler and L. Susskind, Nucl. Phys. B262, 159, (1985).

16. G. 't Hooft, Comm. Math. Phys. 117, 685, (1988).

17. S. Deser and R. Jackiw, Comm. Math. Phys. 118, 495, (1988). 
18. T. Jacobson and L. Smolin, Nucl. Phys. B299, 295, (1988); Class. Quantum Grav. 5, $583,(1988)$.

19. C. Rovelli and L. Smolin, Phys. Rev. Lett. 61, 1155, (1988); Nucl. Phys. B331, 80, (1990).

20. A. Ashtekar, Phys. Rev. D36, 1587, (1987).

21. A. Ashtekar, V. Husain, C. Rovelli, J. Samuel and L. Smolin, Class. Quant. Grav. 6, L185, (1989).

22. S. P. Martin, Nucl. Phys. B327, 178, (1989).

23. J. Nelson and T. Regge, Nucl. Phys. B328, 190, (1989). 


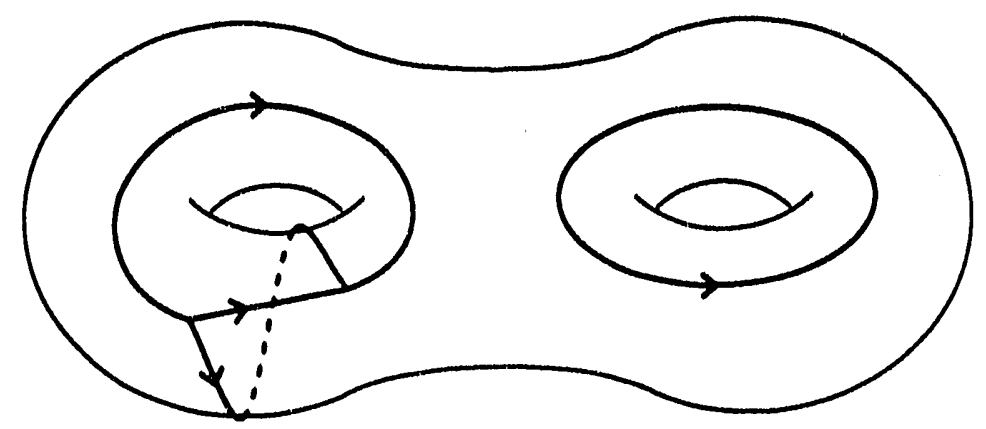

Fig. 1: Two classical canonical graph observables which do not intersect in the spatial manifold must have a vanishing Poisson bracket.

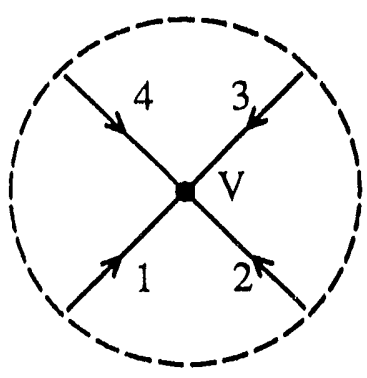

(a)

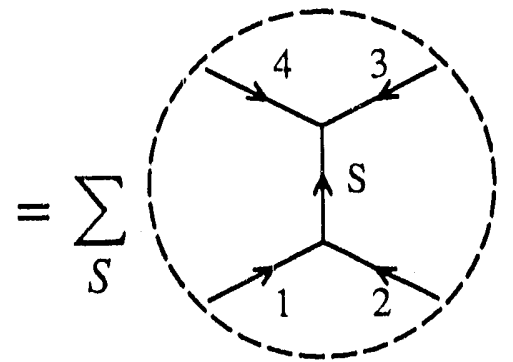

(b)

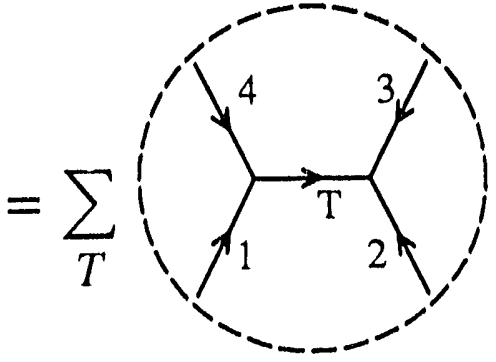

(c)

Fig. 2: All 4-valent vertices (a) can be eliminated in favor of 3-valent vertices as indicated schematically as in (b), or as in (c).

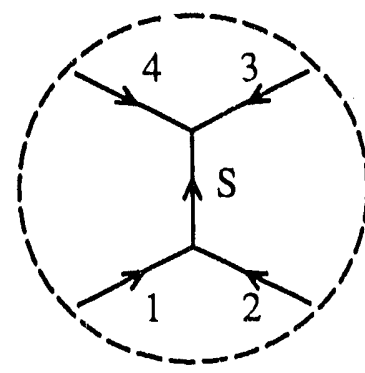

$=\sum_{T}$

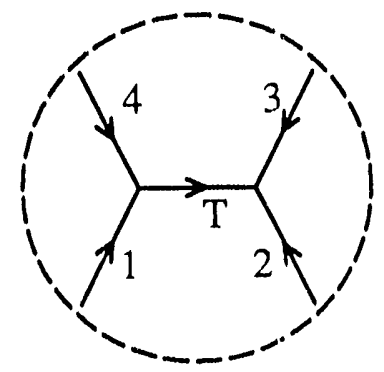

Fig. 3: A schematic of a redundancy relation between graphs with 3-vertices. 


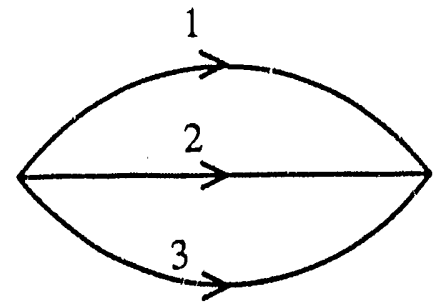

Fig. 4: This canonical graph observable can be deformed to a point and so is just equal to a number.
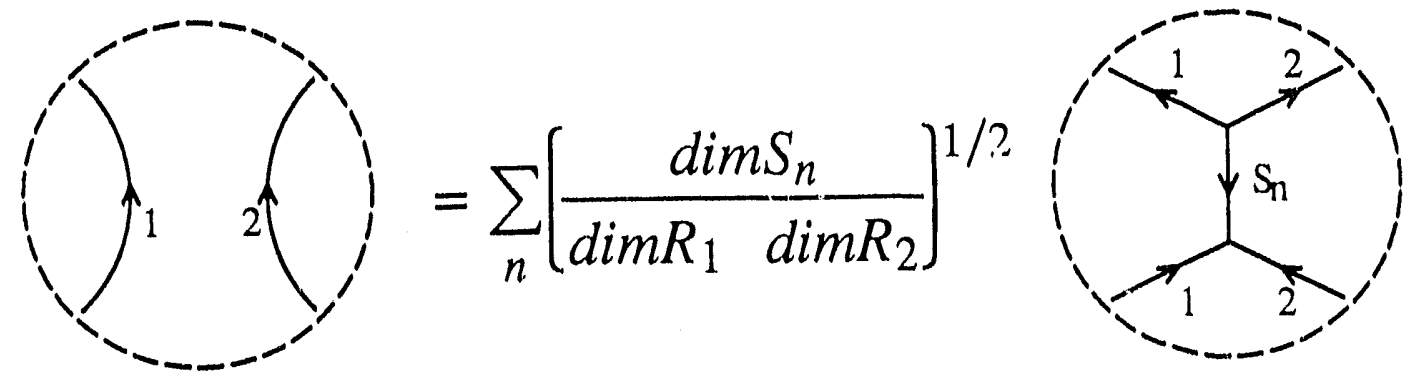

Fig. 5: A redundancy rule which relates different classical graphs which are the same outside of a small neighborhood.

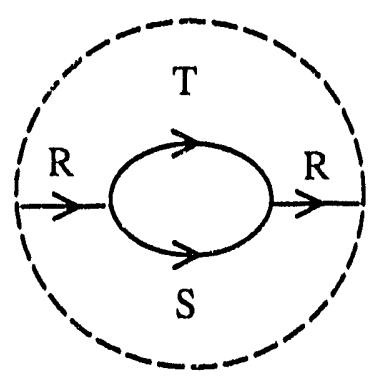

$$
=\left(\frac{\operatorname{dim} S \operatorname{dim} T}{\operatorname{dim} R}\right)^{1 / 2}
$$

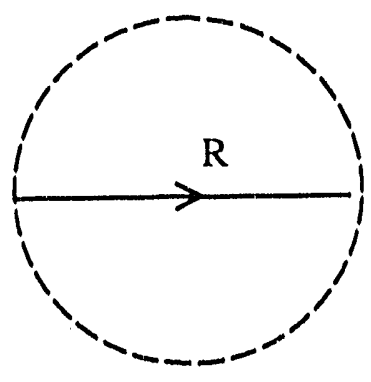

Fig. 6: A redundancy rule which allows us to excise a trivial part of a classical canonical graph observable. 


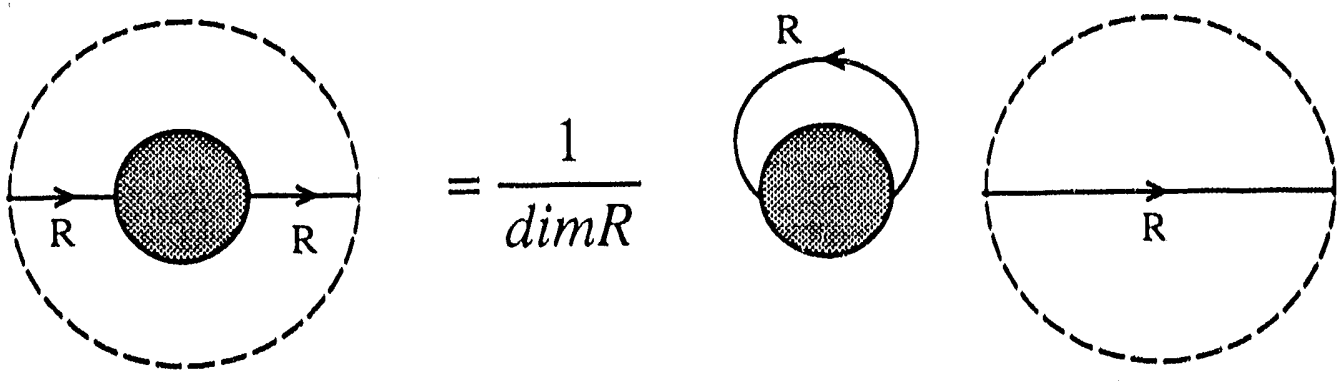

Fig. 7: A trivial part of a classical graph (one that can be deformed to a po.nt in the spatial manifold) can be excised as shown here.

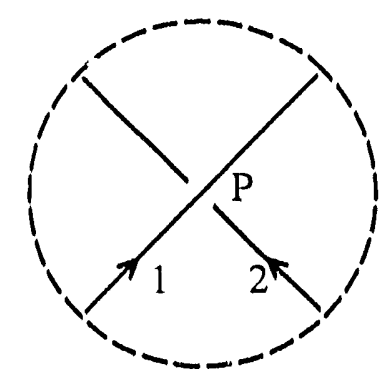

Fig. 8: We wish to compute the Poisson bracket of two canonical graphs which intersect at the point $P$.

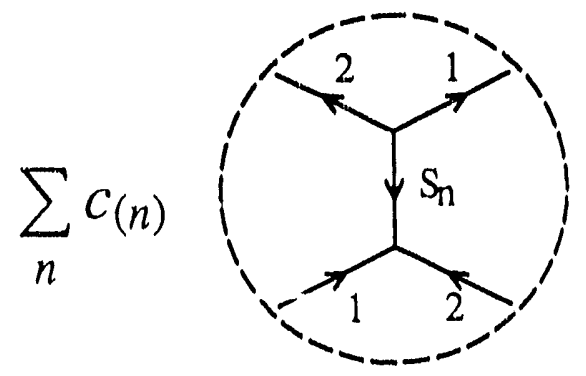

Fig. 9: The contribution to the Poisson bracket of two canonical graphs from the i.ntersection point $\mathrm{P}$ is given by the sum of graphs indicated. 


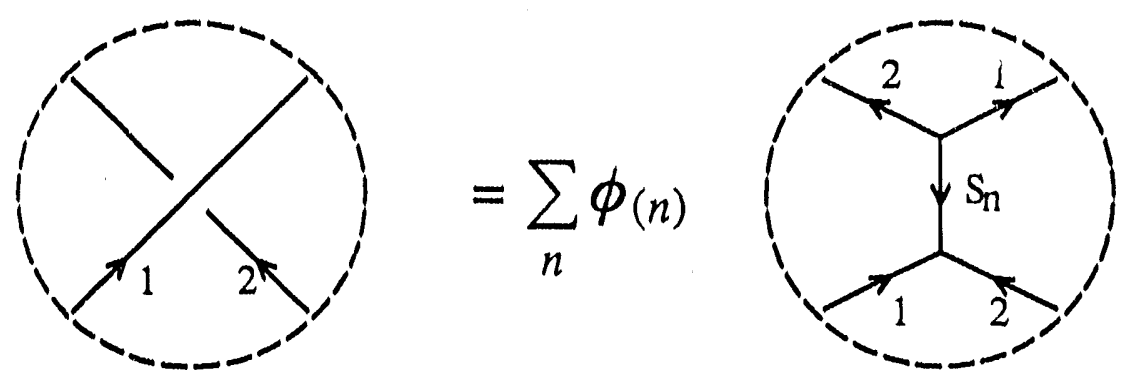

Fig. 10: This skein relation, together with that of Fig. 11, can be used to infer the commutator of two canonical graph observables.

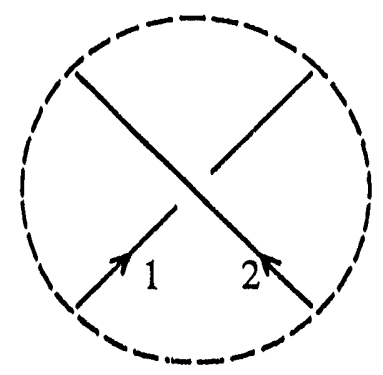

$$
=\sum_{n} \phi_{(n)}^{*}
$$

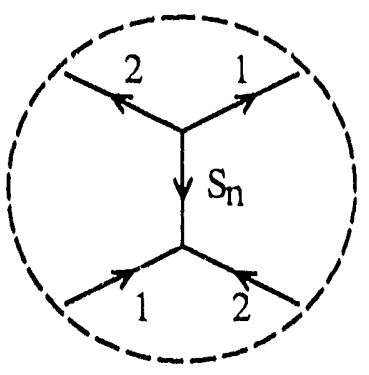

Fig. 11: The skein relation in which the overlap order is reversed from Fig. 10.
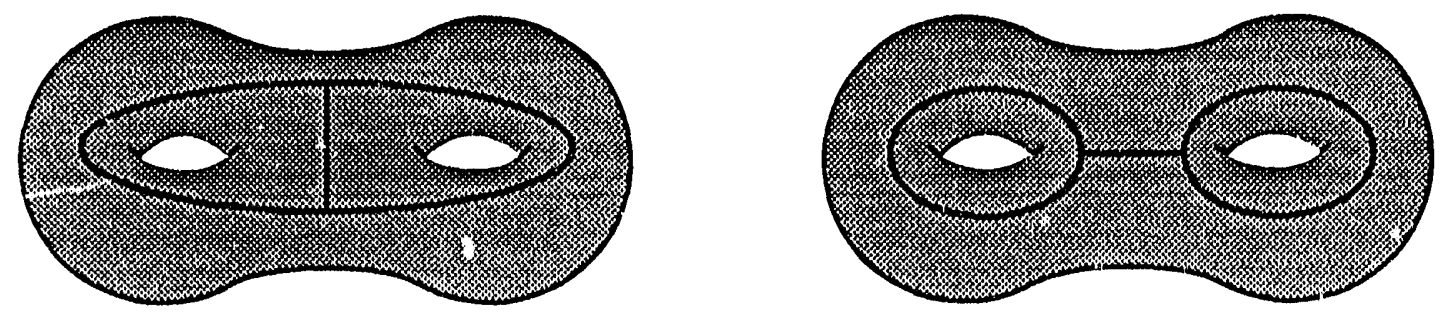

Fig. 12: Two possible choices for the pre-graph at genus 2 . 


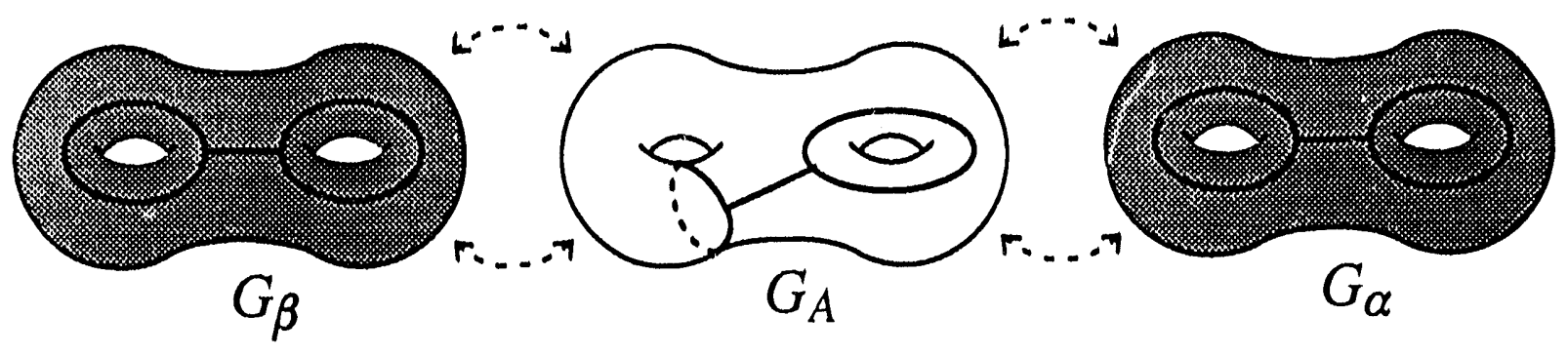

Fig. 13: The matrix element of a canonical graph observable $G_{A}$ between two basis element states $G_{a}$ and $G_{\beta}$ can be computed by gluing together two copies of the solid handlebody.

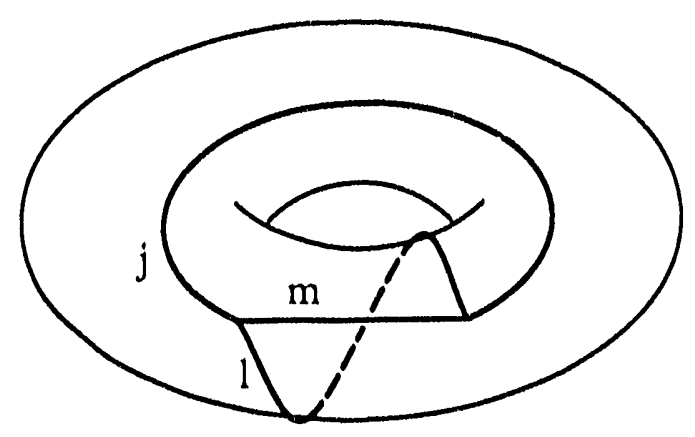

Fig. 14: A graph observable on the torus which arises in the commutator of an a-cycle loop in the spin 1 rep and a b-cycle loop in the spin $j$ rep. 

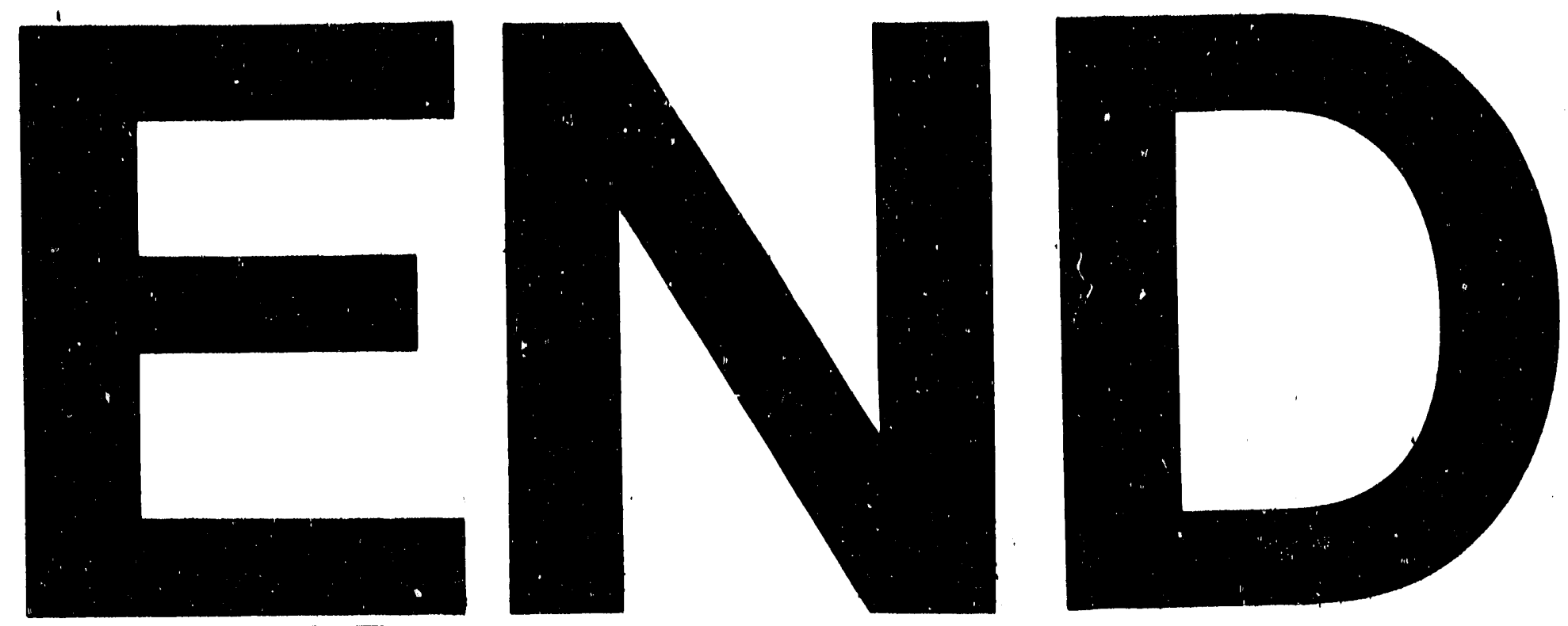

The
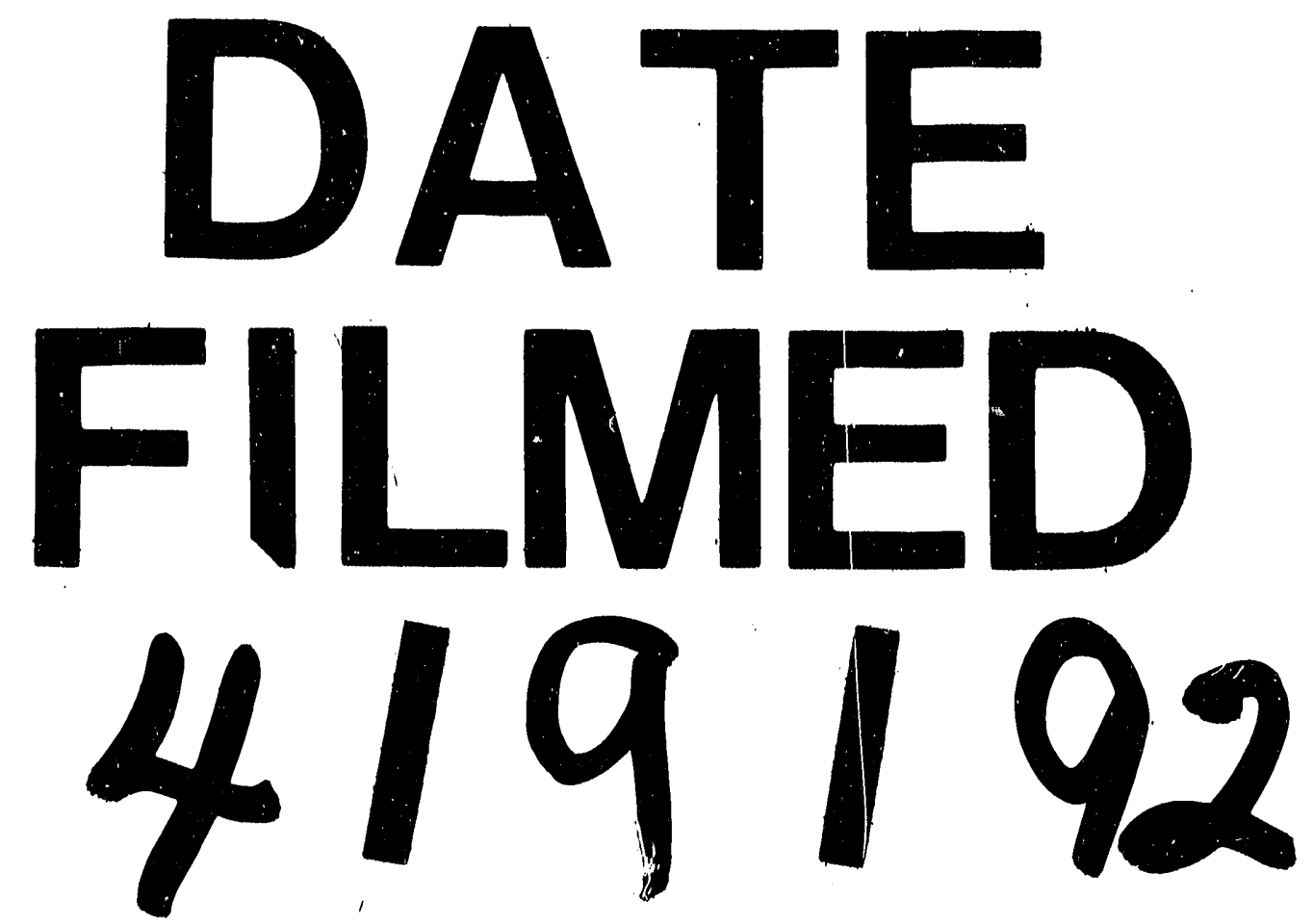

I 
